Journal of Mathematics and Statistics 6 (3): 210-216, 2010

ISSN 1549-3644

(C) 2010 Science Publications

\title{
The Horizontal Electric Field Induced by a Lightning Return Stroke
}

\author{
${ }^{1}$ Scott L. Meredith, ${ }^{1}$ Susan K. Earles and ${ }^{2}$ Niescja E. Turner \\ ${ }^{1}$ Department of Electrical and Computer Engineering, \\ ${ }^{2}$ Department of Physics and Space Sciences, \\ Florida Institute of Technology, Melbourne, Florida, USA
}

\begin{abstract}
Problem statement: Develop a new formula which describes the horizontal electric field induced by a lightning return stroke in contact with an imperfect conductive surface. Approach: A new method for describing the horizontal electric field induced by a lightning return stroke will be presented. The method presented here had utilized an approach which purposely downplayed the physics of how image theory was employed in the presence of an imperfect conductive surface. It did so by adopting a technique which had focused on the geometry that existed between the lightning channel and surface ground. In doing so, new expressions for surface currents had been derived. This study presented the derivation of these currents along with the horizontal electric field which transpired as a result of their usage. Results: The equation derived had elicited the concept that the channel's image varies with surface conductivity. Conclusion: A method for deriving the horizontal electric field induced by a lightning return stroke had been presented. As the results had shown, once the surface conductivity began to decrease, the horizontal electric field played an increasingly more significant role.
\end{abstract}

Key words: Horizontal electric field, degraded image, lightning and imperfect conductors

\section{INTRODUCTION}

Lightning poses a major problem to the world's technological infrastructure due to its reliance upon electronics which are extremely susceptible to both direct and indirect effect strikes. Direct lightning strikes can cause considerable damage upon striking an object given the tremendous amount of current they carry. Some of the entities commonly affected include: personal electronics, power supply generators, commercial buildings and residential structures.

Lightning generates additional and even more elusive constituents that can wreak havoc upon modern electronics. These indirect effects from lightning strikes do not pose much of a physical hazard to people but can cause considerable damage to sensitive electronic components. The electromagnetic fields which propagate outward from the return stroke can couple into sensitive electronic components. Once this occurs, secondary voltage and current constituents add to those already present. This can induce large transient spikes which can lead to catastrophic and/or latent failures to the components or systems they affect.
Given the impact lightning strikes pose to life and property, considerable research has been done in order to establish a better understanding of how they develop and identify all of their effects. Their are four classification of lightning return-stroke models defined by Rakov and Uman (1998), which address the latter, to determine and categorize the electromagnetic constituents induced by a lightning strike. These classes of models include: "Gas dynamic" or "physical" models, "electromagnetic" models, "distributed-circuit" models and "engineering" models. The adopted engineering class of model, places an emphasis upon establishing a close balance between model-predicted electromagnetic fields and those observed at various distances. This study will leverage a type of engineering model to derive the horizontal electric field which transpires from a lightning return stroke in contact with an imperfect conductive surface.

\section{MATERIALS AND METHODS}

Considerable research has been spent treating the surface in contact with the lightning channel as a perfect conductor. This ideology, in turn leveraged image theory to derive the resulting electromagnetic

Corresponding Author: Scott L. Meredith, Department of Electrical and Computer Engineering, Florida Institute of Technology, Melbourne, Florida, USA 
fields. However, recently more emphasis has been placed upon taking into account surfaces which are no longer considered perfect conductors.

In the literature, methods which are typically used to account for the lossy nature of conductive grounds have adopted the wavetilt formula, the Cooray formula and the Cooray-Rubinstein formula. The wavetilt formula relates the Fourier transform of the horizontal electric field to that of the vertical electric field with the following expression (Master and Uman, 1984):

$$
W(j \omega)=\frac{E_{H}(j \omega)}{E_{v}(j \omega)}=\frac{1}{\sqrt{\varepsilon_{r}+\sigma / j \omega \varepsilon_{0}}}
$$

where, $E_{H}(j \omega)$ and $E_{V}(j \omega)$ are the Fourier transforms of the horizontal and vertical electric fields respectively, with the relative permittivity of the soil $\varepsilon_{\mathrm{r}}$, soil conductivity $\sigma$, the imaginary constant $j$, the angular frequency $\omega$ and permittivity of the air $\varepsilon_{0}$. Although, this formula was found to be appropriate for remote observation points, it was later shown to be inapplicable for relatively close ranges (Shoory et al., 2005).

As pointed out by Rubinstein (1996) and Weyl (1919) expressed the results of the Sommerfeld integrals for the fields from a dipole over an imperfectly conductive surface as a group of plane waves that are reflected and refracted by the ground surface at incident angles with both real and imaginary constituents. Rubinstein (1996) goes on to mention that if the surface ground is a relatively good conductor, these plane waves will refract at an angle that is approximately perpendicular to the surface of incident.

We know from image theory that a charge over an infinitely conductive ground has a perfect mirror image. This "mirror image" can be quantified by taking the charge's spatial coordinates which are perpendicular to the surface and rotating or projecting them by $180^{\circ}$ (Balanis, 1989). Taking the cosine of this angle gives rise to an image charge that is equal in magnitude but opposite in polarity. However, in reality the surface in the presence of a charged particle is not a perfect conductor. With this in mind, one must presume that formulas which leverage this "perfect conductor" assumption will loss accuracy as the surface becomes increasing non-conductive. For the purposes of this study, we introduce and leverage an approach proposed by Meredith et al. (2010) to derive the equation for the horizontal electric field which transpires from a lightning return stroke.

Rubinstein (1996) introduced a new formula, presently known as the Cooray-Rubinstein formula that calculates the horizontal electric field above an imperfect conductor. This formula is broken into two terms, both of which assume a perfect conductive ground as shown by:

$$
\begin{gathered}
E_{r}(z=h, r)=E_{\text {rp }}(z=h, r)-H_{\Phi_{p}}(z=0, r) \\
\frac{\sqrt{\mu_{0}}}{\sqrt{\varepsilon_{0}+\sigma / j \omega}}
\end{gathered}
$$

where the first term is the horizontal electric field at a specified height $\mathrm{h}$, the second term is the horizontal magnetic field at ground level multiplied by the surface impedance and the subscript $\mathrm{p}$ denotes a perfect conductor. Rubinstein (1996) goes onto show that for large values of $\mathrm{r}$, (2) reduces to the wavetilt formula. However, it was later shown by Shoory et al. (2005) that the Rubinstein-Cooray formula for calculating the horizontal electric field is a valid approximation for close ranges but becomes inadequate for far ranges and poorly conducting grounds.

General theory: In differential form Maxwell's equations for a homogeneous, time variant and linear medium can be written (Jackson, 1998), where D is the electric displacement, $\mathrm{E}$ is the electric field, $\mathrm{B}$ is the magnetic field, $\mathrm{H}$ is the magnetic field strength, $\mathrm{J}$ is the current density, $\rho$ is the charge distribution per unit volume, $\mu_{0}$ the magnetic permeability and $\varepsilon_{0}$ is the electric permittivity:

$\nabla \cdot \mathrm{E}=\rho / \varepsilon_{0}$

$\nabla \times \mathrm{E}=-\mu_{0} \frac{\partial \mathrm{H}}{\partial \mathrm{t}}$

$\nabla \times \mathrm{H}=\mathrm{J}+\varepsilon_{0} \frac{\partial \mathrm{E}}{\partial \mathrm{t}}$

$\nabla \cdot \mu_{0} \mathrm{H}=0$

The preferred method, the dipole technique, leverages Maxwell's equations to form a system of seven differential equations and seven unknowns given a known current distribution. From (3) through (5) the seven equations can be written as follows:

$$
\begin{aligned}
& \frac{\partial \mathrm{E}_{\mathrm{x}}}{\partial \mathrm{x}}+\frac{\partial \mathrm{E}_{\mathrm{y}}}{\partial \mathrm{y}}+\frac{\partial \mathrm{E}_{\mathrm{z}}}{\partial \mathrm{z}}=\frac{\rho}{\varepsilon_{0}} \\
& \frac{\partial \mathrm{E}_{\mathrm{z}}}{\partial \mathrm{y}}-\frac{\partial \mathrm{E}_{\mathrm{y}}}{\partial \mathrm{z}}=-\mu_{0} \frac{\partial \mathrm{H}_{\mathrm{x}}}{\partial \mathrm{t}}
\end{aligned}
$$




$$
\begin{aligned}
& \frac{\partial \mathrm{E}_{\mathrm{y}}}{\partial \mathrm{z}}-\frac{\partial \mathrm{E}_{\mathrm{z}}}{\partial \mathrm{x}}=-\mu_{0} \frac{\partial \mathrm{H}_{\mathrm{y}}}{\partial \mathrm{t}} \\
& \frac{\partial \mathrm{E}_{\mathrm{y}}}{\partial \mathrm{x}}-\frac{\partial \mathrm{E}_{\mathrm{x}}}{\partial \mathrm{y}}=-\mu_{0} \frac{\partial \mathrm{H}_{\mathrm{z}}}{\partial \mathrm{t}} \\
& \frac{\partial \mathrm{H}_{\mathrm{z}}}{\partial \mathrm{y}}-\frac{\partial \mathrm{H}_{\mathrm{y}}}{\partial \mathrm{z}}=\mathrm{J}_{\mathrm{x}}+\varepsilon_{0} \frac{\partial \mathrm{E}_{\mathrm{x}}}{\partial \mathrm{t}} \\
& \frac{\partial \mathrm{H}_{\mathrm{x}}}{\partial \mathrm{z}}-\frac{\partial \mathrm{H}_{\mathrm{z}}}{\partial \mathrm{x}}=\mathrm{J}_{\mathrm{y}}+\varepsilon_{0} \frac{\partial \mathrm{E}_{\mathrm{y}}}{\partial \mathrm{t}} \\
& \frac{\partial \mathrm{H}_{\mathrm{y}}}{\partial \mathrm{x}}-\frac{\partial \mathrm{H}_{\mathrm{x}}}{\partial \mathrm{y}}=\mathrm{J}_{\mathrm{z}}+\varepsilon_{0} \frac{\partial \mathrm{E}_{\mathrm{z}}}{\partial \mathrm{t}}
\end{aligned}
$$

With the seven unknowns given by: $E_{x}, E_{y}, E_{z}, H_{x}$, $\mathrm{H}_{\mathrm{y}}, \mathrm{H}_{\mathrm{z}}$ and $\rho$. Given Eq. 4 and 6, one can solve for the electric and magnetic fields in terms of the vector potential A. After the usage of some substitutions and vector identities one would obtain:

$$
\mathrm{E}=-\nabla \Phi-\frac{\partial \mathrm{A}}{\partial \mathrm{t}}
$$

and

$$
\mu_{0} \mathrm{H}=\nabla \times \mathrm{A}
$$

Given Lorentz condition:

$$
\nabla \cdot A+\varepsilon_{0} \mu_{0} \frac{\partial \Phi}{\partial t}=0
$$

one can solve for the potential $\Phi$, to obtain:

$$
\Phi(\mathrm{r}, \mathrm{t})=-\frac{1}{\varepsilon_{0} \mu_{0}} \int_{0^{-}}^{\mathrm{t}}(\nabla \cdot \mathrm{A}) \mathrm{d} \tau+\Phi\left(\mathrm{t}=0^{-}\right)
$$

Since at $\mathrm{t}=0^{-}$there is no charge, the scalar potential must be zero as well. Therefore one can write the scalar potential in terms of the vector potential alone whereby:

$$
\Phi(\mathrm{r}, \mathrm{t})=-\mathrm{c}^{2} \int_{0^{-}}^{\mathrm{t}}(\nabla \cdot \mathrm{A}) \mathrm{d} \tau
$$

with $c=\frac{1}{\sqrt{\mu_{0} \varepsilon_{0}}}$ and $c$ equals the speed of light. Next, substitute (18) into (14) to yield:
$\mathrm{E}=\mathrm{c}^{2} \int_{0^{-}}^{\mathrm{t}} \nabla(\nabla \cdot \mathrm{A}) \mathrm{d} \tau-\frac{\partial \mathrm{A}}{\partial \mathrm{t}}$

Knowing the potential A only has a $\mathrm{z}$ component, we can now apply the curl operator in Cartesian coordinates for Eq. 19 to develop an expression for the E-field such that:

$$
E=c^{2} \int_{0^{-}}^{t}\left(\frac{\partial^{2} A_{z}}{\partial z \partial r} i_{r}+\frac{1}{r} \frac{\partial^{2} A_{z}}{\partial \varphi \partial z} i_{\varphi}+\frac{\partial^{2} A_{z}}{\partial z^{2}} i_{z}\right) d \tau-\frac{\partial A_{z}}{\partial t} i_{z}
$$

Due to radial symmetry, the second term in (20) vanishes and one obtains:

$$
E_{r}=c^{2} \int_{0^{-}}^{t} \frac{\partial^{2} A_{z}}{\partial z \partial r} d \tau
$$

and

$$
\mathrm{E}_{\mathrm{z}}=\mathrm{c}^{2} \int_{0^{-}}^{\mathrm{t}} \frac{\partial^{2} \mathrm{~A}_{\mathrm{z}}}{\partial \mathrm{z}^{2}} \mathrm{~d} \tau-\frac{\partial \mathrm{A}_{\mathrm{z}}}{\partial \mathrm{t}}
$$

However, for the purposes of this study, we are only concerned with developing the horizontal electric field. Thus, we shall forgo using (22) and only evaluate (21) which will be used to express the electric field along the coordinate $\mathrm{r}$. We can now use this equation along with the vector potential A:

$$
A=\frac{\mu_{0}}{4 \pi} \int_{v^{\prime}} \frac{J\left(r_{s}, t-R / c\right) d v^{\prime}}{R}
$$

and current distribution:

$i\left(z^{\prime}, t\right)=I_{0} u\left(t-\left|z^{\prime}\right| / v\right)$

where, $\mathrm{u}(\mathrm{t})$ is the Heaviside function, to develop the expression used to describe the horizontal electric field. In doing so, we can now describe the field with:

$$
\begin{aligned}
\mathrm{dE}_{\mathrm{r}}= & \frac{\mathrm{I}_{0} \mathrm{dz} \mathrm{z}^{\prime}}{4 \pi \varepsilon_{0}}\left[\frac{3 r\left(\mathrm{z}-\mathrm{z}^{\prime}\right)}{\mathrm{R}^{5}}\left(\mathrm{t}-\mathrm{R} / \mathrm{c}-\left|\mathrm{z}^{\prime}\right| / \mathrm{v}\right)\right. \\
& \cdot \mathrm{u}\left(\mathrm{t}-\mathrm{R} / \mathrm{c}-\left|\mathrm{z}^{\prime}\right| / \mathrm{v}\right)+\frac{3 \mathrm{r}\left(\mathrm{z}-\mathrm{z}^{\prime}\right)}{\mathrm{cR^{4 }}} \\
& \cdot \mathrm{u}\left(\mathrm{t}-\mathrm{R} / \mathrm{c}-\left|\mathrm{z}^{\prime}\right| / \mathrm{v}\right)+\frac{\mathrm{r}\left(\mathrm{z}-\mathrm{z}^{\prime}\right)}{\mathrm{c}^{2} \mathrm{R}^{3}} \\
& \left.\cdot \delta\left(\mathrm{t}-\mathrm{R} / \mathrm{c}-\left|\mathrm{z}^{\prime}\right| / \mathrm{v}\right)\right]
\end{aligned}
$$


Upon integrating Eq. 25 from $-\mathrm{h}$ to $\mathrm{h}$ along the $\mathrm{z}=0$ plane, we can obtain a closed-form solution for the horizontal electric field as shown by the following:

$$
\begin{aligned}
& \mathrm{E}_{\mathrm{r}}(\mathrm{r}, 0, \mathrm{t})=\frac{\mathrm{I}_{0}}{2 \pi \varepsilon_{0}}\left[\frac{\mathrm{rt}}{\left(\mathrm{h}^{2}+\mathrm{r}^{2}\right)^{3 / 2}}-\frac{\mathrm{t}}{\mathrm{r}^{2}}+\frac{\mathrm{h}^{3}}{\mathrm{vr}\left(\mathrm{h}^{2}+\mathrm{r}^{2}\right)^{3 / 2}}\right. \\
& \left.-\frac{\mathrm{rh}}{\mathrm{c}^{2}\left(\mathrm{~h}^{2}+\mathrm{r}^{2}\right)^{3 / 2}\left(\frac{1}{\mathrm{v}}+\frac{\mathrm{h}}{\mathrm{c} \sqrt{\mathrm{h}^{2}+\mathrm{r}^{2}}}\right)}\right]
\end{aligned}
$$

The degraded image: In order to augment the approach used from image theory, this study will introduce the idea of the degraded image (Meredith et al., 2010). This ideology accommodates both perfect and imperfect projected images. With traditional image theory, it assumes that a charge in the presence of a perfect conductor has a mirror image as depicted in Fig. 1.

However, as the surface in the presence of a charged particle becomes less conductive, one can no longer assume that the image remains unchanged. In fact, one must concede to the idea that the charge's image can no longer be projected in the same fashion as the image of a charge in the presence of a perfect conductor. With the adoption of this idea in place, it's logical to presume that as the grounding surface becomes less conductive, the image will become degraded. Meredith et al. (2010) surmised that the image charge's vertical position can vary as a function of surface conductivity. This methodology greatly simplified how image theory could be employed for surfaces which are no longer consider perfect conductors. In doing so, a geometric relationship between the degraded charge and surface conductivity could be established. This methodology is illustrated in Fig. 2 and 3.

Figure 2 illustrates the how the degraded image charge $q_{d}$, will change with respect to the perfect image charge $\mathrm{q}_{\mathrm{p}}$, as the conductivity $\sigma$, of the surface decreases. Figure 3 illustrates, the contribution from the degraded charge can be quantified by taking the magnitude of the perfect image charge and scaling it by a factor which accounts for the loss. This can be realized by taking the projection of the perfect image's magnitude and rotating it along the $\mathrm{z}=0$ axis until it shares the same $z$ component as the degraded image. Doing so will allow one to associate the perfect image with the degraded image by multiplying the scaling factor, $\cos (\gamma)$.

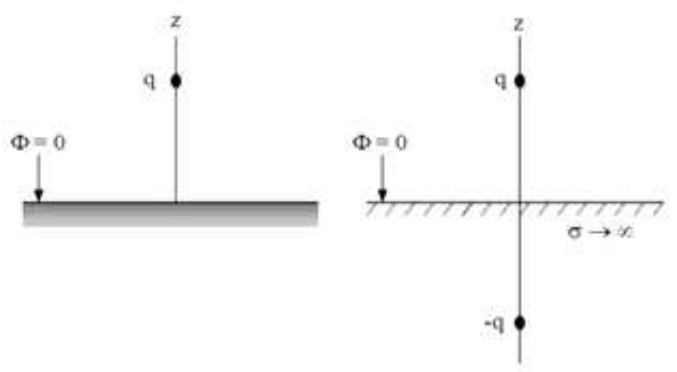

Fig. 1: Solution by method of images

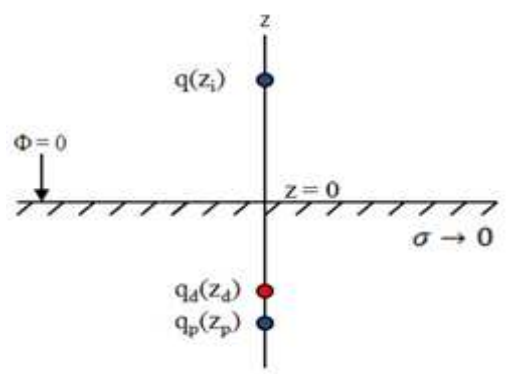

Fig. 2: Application of the degraded image

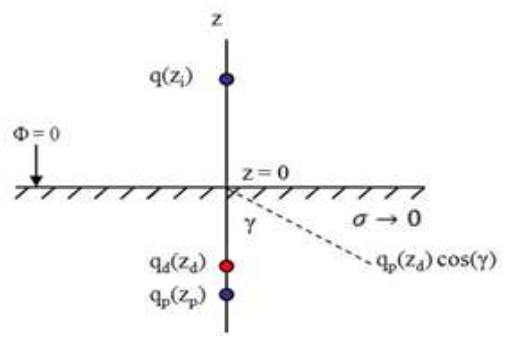

Fig. 3: The projection of a perfect image's magnitude

We can now extend this idea to encompass the geometry which exists between the lightning channel and surface ground. If we assume that the lightning channel's width is finite, then any degradation to the channel will only occur along the z-axis. Given the image channel has become degraded, some of the charges that were once part of this channel have become displaced. Since the conservation of charge must be preserved, these dislocated charges will now collect along the surface and induce currents which travel away from the channel. As a consequence, the image current now contributes less to the vertical electric and azimuthal magnetic fields while creating a horizontal electric field.

Figure 4 illustrates the how the degraded image will change with respect to the perfect image as the conductivity $\sigma$, of the surface approaches zero. 


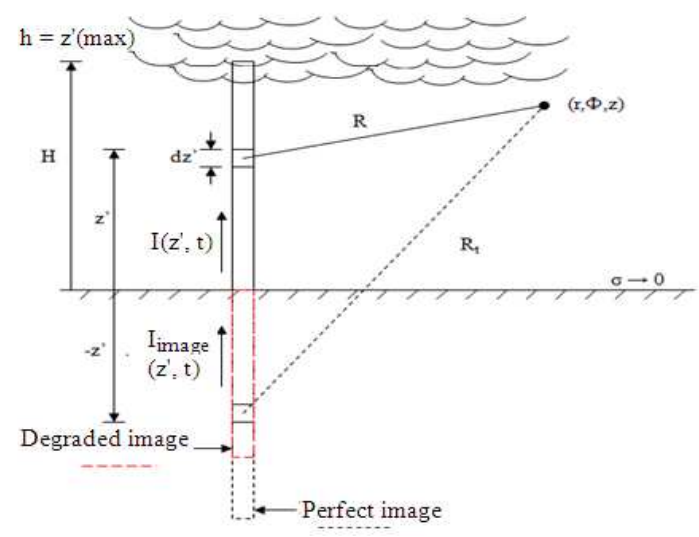

Fig. 4: Application of the degraded image used for lightning channel

Once the surface becomes a perfect insulator, the magnitude of the degraded image channel will equal zero. The methodology used here purposely downplays the physics of how image theory is employed to account for a charge which is in the presence of an imperfect conductive surface. In doing so, the proposed method formulates a solution that has minimized the complexity of the original problem while providing an approximation founded upon a geometric relationship. Knowing how the image channel is affected by the surface conductivity allows one to develop an equivalency between the two by exploiting the geometry of Fig. 4. Since the magnitude of the perfect image current is generally known, this value can be scaled to account for the change in conductivity.

As Fig. 5 illustrates, the contribution from the degraded image can be quantified by taking the magnitude of the current which travels along the perfect image and scaling by a factor which accounts for the loss. This can be achieved by taking the projection of the perfect image current and rotating it along the $\mathrm{r}-\mathrm{z}$ axis until it shares the same $\mathrm{z}$ component as the degraded image. Doing so will allow one to associate the perfect image with the degraded image by multiplying the scaling factor, $\cos (\gamma)$. Therefore, we can write the degraded current in terms of the perfect current such that:

$$
I_{d}=I_{0} \cos (\gamma)
$$

Where:

$\mathrm{I}_{\mathrm{d}}=$ The degraded imaged current

$\mathrm{I}_{0}=$ The perfect image current

$\gamma=$ The angle between the perfect image and degraded image

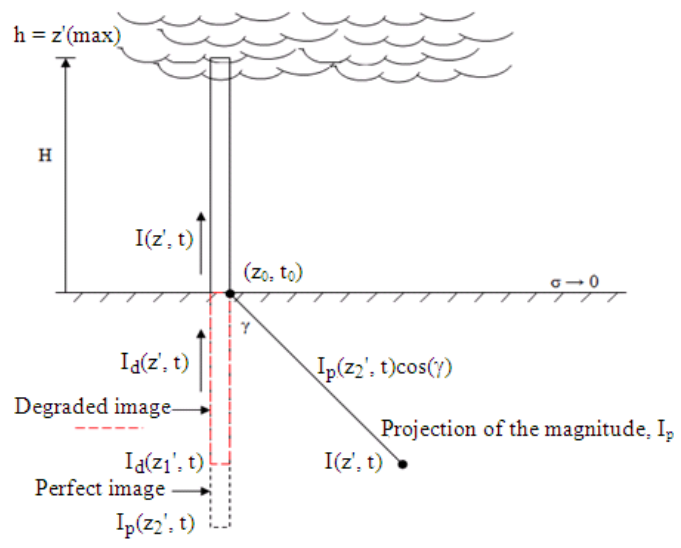

Fig. 5: Projection of the degraded current used from the lightning channel

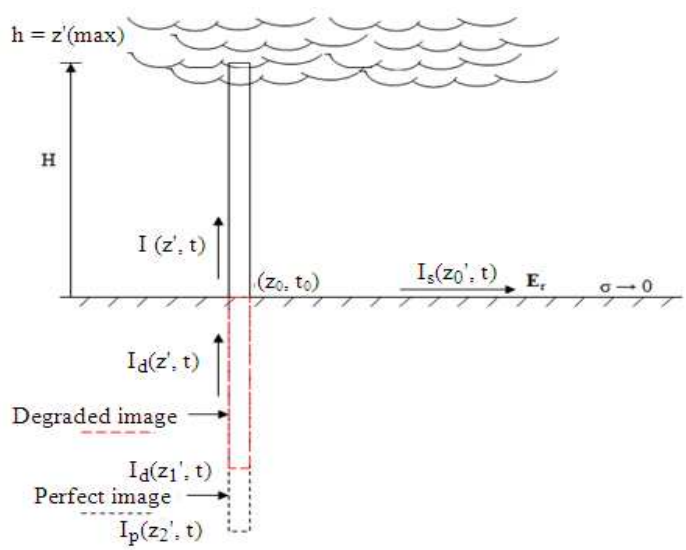

Fig. 6: Surface current traveling away from the lightning channel

In order to maintain the conservation of charge, one must arrive at the notion that the imaged charge constituents that are no longer present within the degraded image channel must now be present elsewhere. With this in mind, we can infer that these imaged charges collect along the surface and induce currents which create electromagnetic fields in addition to those originally accounted for (Rubinstein and Uman, 1989). Although these surface currents spread out radially, their contributions can be approximated by formulating two distinct currents, each of which lie on opposite sides of the channel-ground interface. In principle, these two surface currents represent the summation of each of their respective radial constituents, thus formulating a viable approximation to the currents present. Figure 6 illustrates one of the two surface currents which travel away from the lightning 
channel. We can now describe each of these surface currents with the following:

$$
\mathrm{I}_{\mathrm{s}}=\frac{\mathrm{I}_{0}}{2}(1-\cos (\gamma))
$$

where, $I_{s}$ equals the surface current on either side of the lightning channel. By summing (27) and (28) we can now describe the contributions made by the imaged current for both perfect and imperfect conductive surfaces. The total imaged current can now be written as:

$$
I_{I}=I_{0} \cdot \cos (\gamma)+\frac{I_{0}}{2}(1-\cos (\gamma))
$$

where, $I_{I}$ equals the imaged current from the degraded image current and surface current on either side of the lightning channel.

\section{RESULTS}

In general, the surface in contact with the lightning channel is presumed to be perfect whereby resulting in a horizontal electric field which equals zero. However, as the surface in contact with the return stoke becomes less conductive, this field's magnitude is no longer negligible. Equation 26 depicts the electric field which traditionally travels along the coordinate $\mathrm{r}$. Its usage is negated when a perfect conductive model is used. However, it plays an increasingly more significant role once this model is no longer valid. Given the expressions (27-29) account for surfaces of varying conductivities, allows one to now augment (26) to account for imperfect conductors. Thus, we can rewrite (26) to account for varying types of conductive surfaces such that:

$$
\begin{gathered}
E_{r}(r, 0, t)=\frac{I_{s}}{2 \pi \varepsilon_{0}}\left[\frac{\mathrm{rt}}{\left(h^{2}+r^{2}\right)^{3 / 2}}-\frac{t}{r^{2}}+\frac{h^{3}}{v r\left(h^{2}+r^{2}\right)^{3 / 2}}\right. \\
\left.-\frac{r h}{c^{2}\left(h^{2}+r^{2}\right)^{3 / 2}\left(\frac{1}{v}+\frac{h}{c \sqrt{h^{2}+r^{2}}}\right)}\right]
\end{gathered}
$$

One can now utilize (30) to graphically illustrate how the magnitudes of the horizontal electric fields change over time with varying surface conductivity with the Fig. 7 and 8.

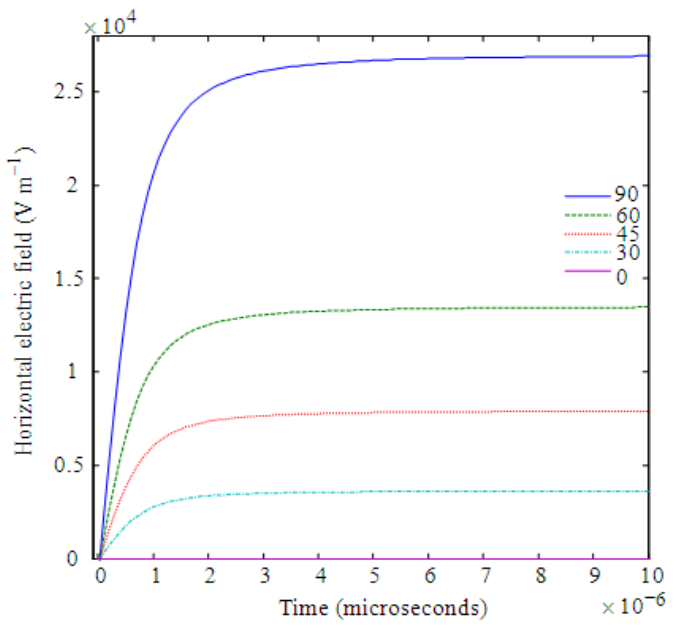

Fig. 7: Illustration of the horizontal electric fields induced by lightning striking varying types of conductive surfaces when $r=100 \mathrm{~m}$

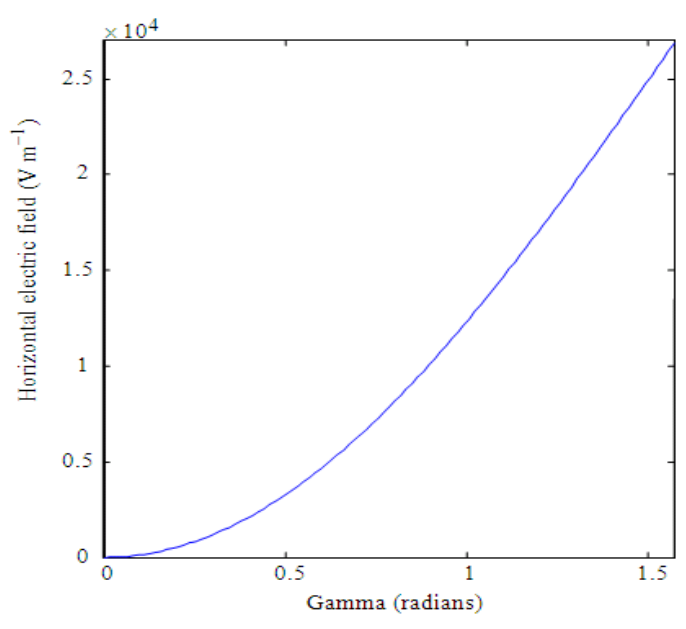

Fig. 8: Illustration of the horizontal electric field when $\mathrm{t}=1 \times 10^{-5}$ over the interval $(0, \mathrm{pi} / 2)$

As Fig. 7 shows, the effect to the horizontal electric field when $\gamma=0,30,45,60$ and $90^{\circ}$ is appreciable. One readily observes that as the angle gamma begins to increase, the subsequent horizontal electric field will increase as well.

Figure 8 shows how the horizontal electric field varies as a result of surface conductivity at a fixed time $t$.

\section{DISCUSSION}

In Eq. 30, we modified the current waveform to reflect how the lightning channel's image changes with surface conductivity. This methodology has greatly 
simplified how image theory was employed for surfaces which are no longer consider perfect conductors. In doing so, an alternative method for deriving the horizontal electric field induced by a lightning return stroke has been presented.

\section{CONCLUSION}

A new method for describing the horizontal electric field which originates from a lightning return stroke in contact with perfect and imperfect conductive surfaces has been presented. Surmising the image channel's vertical position can vary as a function of surface conductivity aided in the development of the derived formula. This methodology has greatly simplified how image theory could be employed for surfaces which are no longer consider perfect conductors. In doing so, surface currents which transpired as a result of the proposed method gave rise to a horizontal electric field not previously seen in literature. As the results have shown, once the surface conductivity began to decrease, the horizontal electric field played an increasingly more significant role.

\section{REFERENCES}

Balanis, C.A., 1989. Advanced Engineering Electromagnetics. John Wiley and Sons Inc., New Jersey, USA., ISBN: 0-471-62194-3, pp: 314-322.

Jackson, J.D., 1998. Classical Electrodynamics. 3rd Edn., John Wiley and Sons Inc., New Jersey, USA., ISBN: 0-471-30932-X, pp: 808.
Master, M.J. and M.A. Uman, 1984. Lightning induced voltages on power lines: Theory. IEEE Trans. Power Apparat. Syst., PAS-103: 2502-2518.

Meredith, S.L., S.K. Earles and N.E. Turner, 2010. A new method to describe image theory for an imperfect conductor. J. Math. Stat., 6: 131-135.

Rakov, V.A. and M.A. Uman, 1998. Review and evaluation of lightning return stroke models including some aspects of their application. IEEE Trans. Electromag. Capab., 40: 403-426.

Rubinstein, M. and M.A. Uman, 1989. Methods for calculating the electromagnetic fields from a known source distribution: Application to lightning. IEEE Trans. Electromag. Compat., 31: 183-189.

Rubinstein, M., 1996. An approximate formula for the calculation of the horizontal electric field from a lightning at close, intermediate and long range. IEEE Trans. Electromag. Compat., 38: 531-535.

Shoory, A., R. Moini, S.H. Sadeghi and V.A. Rakov, 2005. Analysis of lightning-radiated electromagnetic fields in the vicinity of lossy ground. IEEE Trans. Electromag. Compat., 47: 131-145.

Weyl, H., 1919. Ausbreitung elektromagnetischer wellen über einer ebenen leiter. Ann. D. Physik, 60: 481-500. http://zs.thulb.unijena.de/receive/jportal_jparticle_00128178;jsession $\mathrm{id}=$ F73A581692B7CC751FCD3B7ED0BCED93?1 ang=en 